\title{
Effect of Neutral Current Interactions on High Energy Muon and Electron Neutrino Propagation through the Earth
}

\author{
Annalisa L'Abbate ${ }^{\mathrm{a}}$, Teresa Montaruli ${ }^{\mathrm{a}, \mathrm{b}}$, Igor Sokalski ${ }^{\mathrm{b}, \mathrm{c}, *}$ \\ ${ }^{a}$ Physics Department, University of Bari, Via Amendola 173, I-70126 Bari, Italy \\ ${ }^{\mathrm{b}}$ Istituto Nazionale di Fisica Nucleare / Sezione di Bari, Via Amendola 173, \\ I-70126 Bari, Italy \\ ${ }^{\mathrm{c}}$ Institute for Nuclear Research of the Russian Academy of Sciences, 60th October \\ Anniversary Prospect 7a, RU-117312 Moscow, Russia
}

\begin{abstract}
High energy $\nu_{\mu}$ and $\nu_{e}$ propagation through the Earth has been performed using the Monte Carlo technique. We focused our attention on the effect of neutral current deep inelastic interactions $\nu_{l} N \stackrel{N C}{\longrightarrow} \nu_{l} X$ compared to that of charged current ones $\nu_{l} N \stackrel{C C}{\longrightarrow} l X$. We have found that NCs do not produce any significant effect with respect to the case in which only CCs are considered. Therefore we conclude that $\mathrm{NC}$ interactions can be neglected without considerable loss of accuracy. When computing upward-going neutrino fluxes a simple formula describing the transmission probability, that depends on the neutrino direction and energy and the CC cross section, can be used to account for the Earth shadowing effect.
\end{abstract}

Key words: high energy neutrino, neutrino interactions, charged current, neutral current, Earth, neutrino telescope, Monte Carlo technique

PACS: $02.70 . \mathrm{Uu}, 13.15 .+\mathrm{g}, 14.60 . \mathrm{Lm}, 98.70 . \mathrm{Sa}$

\footnotetext{
* Corresponding author. Phone: +39-080-544-2347, fax: +39-080-544-2470.

Email addresses: labbate@ba.infn.it (Annalisa L'Abbate), montaruli@ba.infn.it (Teresa Montaruli), sokalski@ba.infn.it (Igor Sokalski).
}

Preprint submitted to Elsevier Science 29 October 2018 


\section{Introduction}

Numerous works were published during the last decade on high energy neutrino propagation through the Earth (see, e.g. [1-11], though the whole list of publications dedicated to this item is much longer). This is an important topic since, in order to correctly estimate the event rates due to neutrinos crossing the Earth that interact close or inside underwater neutrino telescopes (UNTs) [12-17], the Earth shadowing effect needs to be accounted for.

The most complex case is that of $\nu_{\tau}$. Besides suffering neutral current (NC) interactions, it undergoes a regeneration chain of processes in the Earth due to charged current (CC) interactions and consequent fast $\tau$-lepton decay, producing a $\nu_{\tau}$ of lower energy than the initial one: $\nu_{\tau} \stackrel{C C}{\longrightarrow} \tau \rightarrow \nu_{\tau} \ldots$. Moreover, secondary $\nu_{e}$ 's and $\nu_{\mu}$ 's are generated via $\tau^{+} \rightarrow \nu_{e} \bar{\nu}_{\tau} e^{+}, \tau^{-} \rightarrow \bar{\nu}_{e} \nu_{\tau} e^{-}$, $\tau^{+} \rightarrow \nu_{\mu} \bar{\nu}_{\tau} \mu^{+}$and $\tau^{-} \rightarrow \bar{\nu}_{\mu} \nu_{\tau} \mu^{-}$, decays. Hence, a $\nu_{\tau}$ with initial energy at Earth of $E_{\nu_{\tau}}^{0}$ leaves it with an energy $E_{\nu_{\tau}}^{1} \leq E_{\nu_{\tau}}^{0}$, being accompanied by some amount of secondary $\nu_{\mu, e}$ 's $[5,8]$.

In contrast to $\nu_{\tau}$ 's, $\nu_{e}$ 's and $\nu_{\mu}$ 's can only loose energy undergoing a $\mathrm{NC}$ interaction $\nu_{l} N \stackrel{N C}{\longrightarrow} \nu_{l} X$ or be absorbed due to a CC interaction $\nu_{l} N \stackrel{C C}{\longrightarrow}$ $l X^{1}$. Generally, to account properly these two processes one has to solve complex transport equations or apply Monte Carlo (MC) simulation. However, if NC interactions are neglected, the neutrino flux emerging from the Earth $\Phi_{1}\left(E_{\nu_{\mu, e}}, \theta\right)$ is given by the following simple formula which depends only on the neutrino energy $E_{\nu_{e, \mu}}$ and nadir angle $\theta$ :

$$
\Phi_{1}\left(E_{\nu_{\mu, e}}, \theta\right)=S\left(E_{\nu_{\mu, e}}, \theta\right) \times \Phi_{0}\left(E_{\nu_{\mu, e}}, \theta\right)
$$

Here $\Phi_{0}\left(E_{\nu_{\mu, e}}, \theta\right)$ is the incident flux and $S\left(E_{\nu_{\mu, e}}, \theta\right)$ is the screening factor (or transmission probability) which is defined as [1]:

$$
S\left(E_{\nu_{\mu, e}}, \theta\right)=1-p_{a b s}\left(E_{\nu_{\mu, e}}, \theta\right)=\exp \left[-N_{A} \times \sigma_{C C}\left(E_{\nu_{\mu, e}}\right) \times \int \rho(\theta, l) d l\right],
$$

where $p_{a b s}\left(E_{\nu_{\mu, e}}, \theta\right)$ is the absorption probability, $\sigma_{C C}\left(E_{\nu_{\mu, e}}\right)$ is the total CC cross section, $N_{A}$ is the Avogadro number, $\rho(\theta, l)$ is the Earth density profile for a given direction $\theta$ and $l$ is the distance in the Earth on which the integral is performed to calculate the column depth.

In this short paper we describe the results of the MC simulations for $\nu_{\mu}$ and

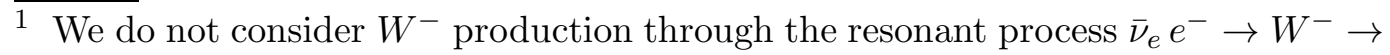
$X$ at $E_{\bar{\nu}_{e}} \approx 6.3 \mathrm{PeV}$ (the Glashow resonance [18]) which occurs only to $\bar{\nu}_{e}$ in a very narrow energy range. 
$\nu_{e}$ propagation through the Earth, focusing on the role of NC interactions and analyzing the accuracy of the formula in Eq. (1).

\section{Method and Results}

The simulation we have used is fully described in [8]. Shortly, we remind that deep inelastic cross sections are calculated using CTEQ3-DIS parton distribution functions [19] taken from PDFLIB library [20]. We have assumed the Earth composition made by standard rock $(A=22, Z=11)$ of variable density with the Earth density profile taken from [21] (Fig. 1).
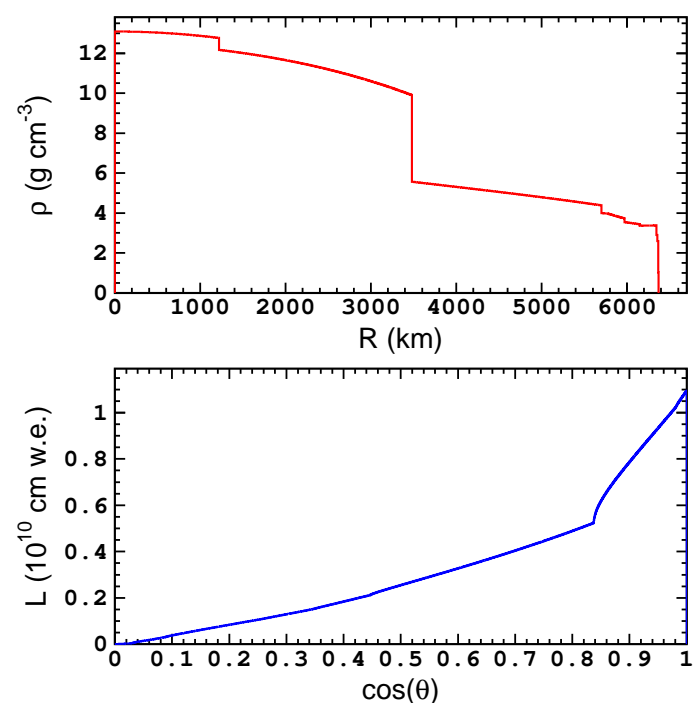

Fig. 1. The Earth density profile [21] (upper panel) and the Earth column depth vs nadir angle (lower panel) as it is used in our calculations.

\subsection{Results on monoenergetic neutrino beams}

We have simulated 1640 monoenergetic neutrino beams each of $5 \times 10^{5}$ events $\left(\nu_{e}, \bar{\nu}_{e}, \nu_{\mu}\right.$ and $\bar{\nu}_{\mu}, 41$ neutrino energies in the range $10 \mathrm{GeV} \leq E_{\nu} \leq 10^{9} \mathrm{GeV}$ and 10 nadir angles in the range $0.1 \leq \cos \theta \leq 1.0)$ and we have saved information on neutrinos which survive after propagation.

The CC cross section $\sigma_{C C}$, that provides the probability of muon and electron neutrino absorption, grows with energy and consequently the interaction length $L_{\text {int }}^{C C}=\left(N_{A} \times \sigma_{C C}\right)^{-1}$ (in water equivalent units) decreases. At some 'transparency' energy $L_{\text {int }}^{C C}$ becomes equal to the Earth column depth seen by the neutrino for a given nadir angle $\theta$ and so, at $E_{\nu_{\mu, e}}>E_{\text {transp }}(\theta)$ the Earth begins to consistently absorb $\nu_{\mathrm{e}, \mu}$ 's. $E_{\text {transp }}(\theta)$ grows with the nadir angle. It 

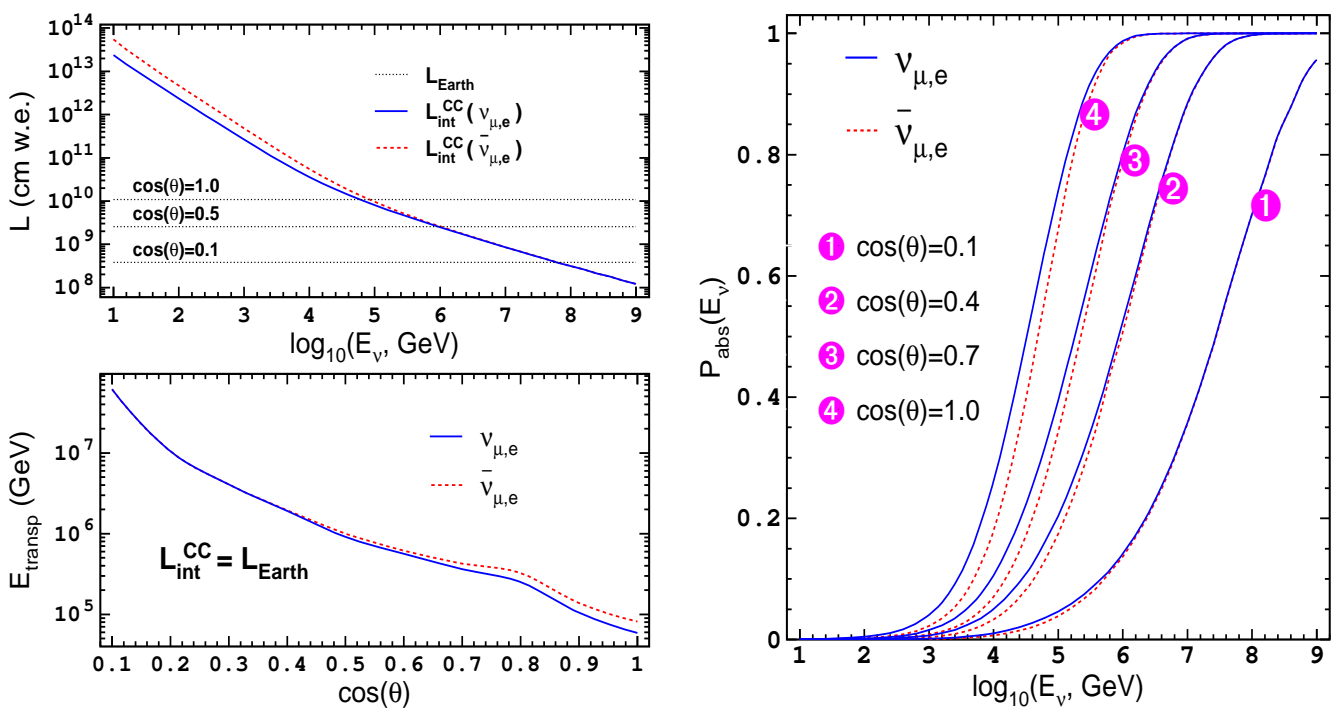

Fig. 2. Left upper panel: CC interaction length vs $E_{\nu_{\mu, e}}$. The three horizontal lines are the Earth column depth for 3 different values of the nadir angle $(\cos \theta=0.1$, $0.5,1.0)$. Left lower panel: transparency energy vs $\cos \theta$. The transparency energy is defined as the energy at which the neutrino interaction length equals the Earth column depth. A 'knee' at $\cos \theta \approx 0.8$ corresponds to an 'ankle' at the curve for the Earth column depth vs nadir angle (Fig. 1) which originates from the fact that the Earth density changes sharply at the distance $\sim 3500 \mathrm{~km}$ from the center. Right panel: absorption probability for $\nu_{\mu, e}$ 's $\left(\bar{\nu}_{\mu, e}\right.$ 's) in the Earth. In the simulation of propagation through the Earth only CC interactions were taken into account. In all panels solid lines are for neutrinos and dashed ones for anti-neutrinos.
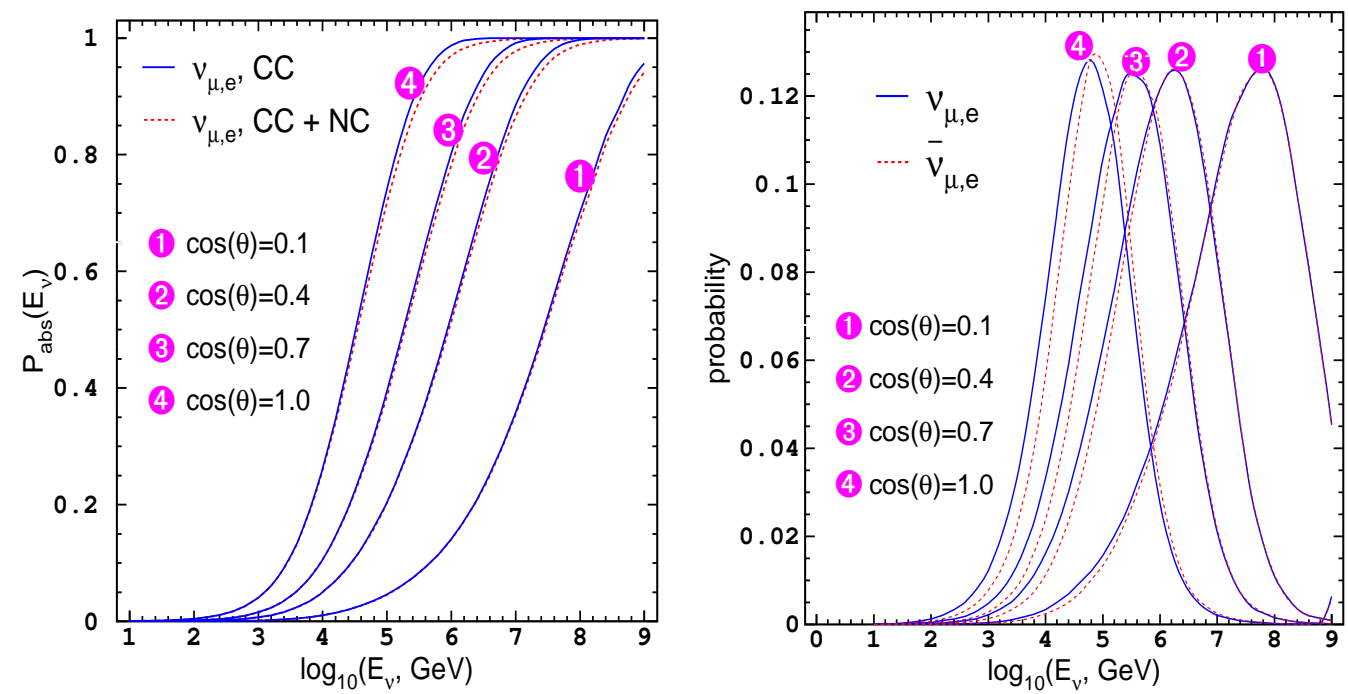

Fig. 3. Left panel: neutrino absorption probability in the Earth taking into account (dashed lines) or not (solid lines) NC interactions. Right panel: probability for $\nu_{\mathrm{e}, \mu}$ 's $\left(\bar{\nu}_{\mathrm{e}, \mu}\right.$ 's) to pass through the Earth with no CC interactions but with at least one $\mathrm{NC}$ interaction (solid lines are for neutrinos and dashed ones for anti-neutrinos). In both plots results are given for four nadir angles $(\cos \theta=0.1,0.4,0.7,1.0)$. 
is about $50-80 \mathrm{TeV}$ at directions close to the vertical $\left(\theta=0^{\circ}\right)$, it becomes about $60 \mathrm{PeV}$ at $\cos (\theta)=0.1\left(\theta=84^{\circ}\right)$ and increases to larger values for more horizontal directions since the matter to be transversed decreases (Fig. 2, left panels). For very horizontal directions the composition of the Cherenkov media where the array is located or also the details of the coast for a specific underwater detector should be known to calculate exactly the transparency energy. Anyway, its value will be very large. Correspondingly, the probability that a neutrino undergoes a CC interaction and is absorbed grows with $E_{\nu_{\mu, e}}$. In the right panel of Fig. 2 the absorption probability $p_{a b s}\left(E_{\nu_{\mu, e}}, \theta\right)$, computed as the ratio between the number of absorbed and propagated neutrinos for a given energy and nadir angle, is shown in the case where only $\mathrm{CC}$ interactions are simulated.

In the considered energy range, $\sigma_{N C}$ is 2.3-3.2 times smaller compared to $\sigma_{C C}$ and hence, the probability that a CC interaction occurs is higher than that of a NC. When $\nu_{\mathrm{e}, \mu}$ 's interact by a NC, the neutrino energy decreases on average by a factor $1-\langle y\rangle=0.5-0.8$ (depending on energy), since the rest of the energy $\langle y\rangle E_{\nu_{\mu, e}}$ is taken by the hadronic shower. Hence, the probability that another $\mathrm{CC}$ interaction occurs decreases. In other words, the probability $p_{a b s}\left(E_{\nu_{\mu, e}}, \theta\right)$ that the neutrino will be absorbed becomes lower. But quantitatively the effect is very small as it can be seen in the left panel of Fig. 3 where $p_{a b s}\left(E_{\nu_{\mu, e}}, \theta\right)$ for $\nu_{\mathrm{e}, \mu}$ 's is plotted for propagation in the Earth with- and without accounting for $\mathrm{NC}$ interactions (results for $\bar{\nu}_{\mathrm{e}, \mu}$ 's are qualitatively the same). Comparing the $\mathrm{NC}+\mathrm{CC}$ case to the $\mathrm{CC}$ only case, we observe only a slight decrease of the absorption probability. The right panel of Fig. 3 shows the probability $p_{N C}$ that $\nu_{\mathrm{e}, \mu}$ 's survive after crossing the Earth with no $\mathrm{CC}$ interactions but at least one $\mathrm{NC}$ interaction. The maximum value of $p_{N C}$ for different neutrino nadir angles is about 0.13 for energies around the transparency energy $E_{\text {transp }}(\theta)$. At lower energies it falls down due to the cross section $\sigma_{N C}$ almost linear decrease with energy. At higher energies it is suppressed due to the increase of $\sigma_{C C}$ since, even after loosing energy in $\mathrm{NC}$ interactions, the neutrino energy remains high enough to undergo another $\mathrm{CC}$ interaction that makes the neutrino disappear. In any case, $p_{N C}$ is remarkably lower compared to $p_{C C}$ and, therefore, it does not affect significantly the total $p_{a b s}\left(E_{\nu_{\mu, e}}, \theta\right)$, which turns out to be very close to $p_{C C}$.

\subsection{Results on various energy spectra}

We have investigated the effect of muon neutrino propagation through the Earth ${ }^{2}$ for various models of neutrino fluxes incident on the Earth when only

$\overline{2}$ All the results and conclusions relevant to $\nu_{\mu}$ 's are also valid for $\nu_{e}$ 's since differential cross sections are practically the same. 

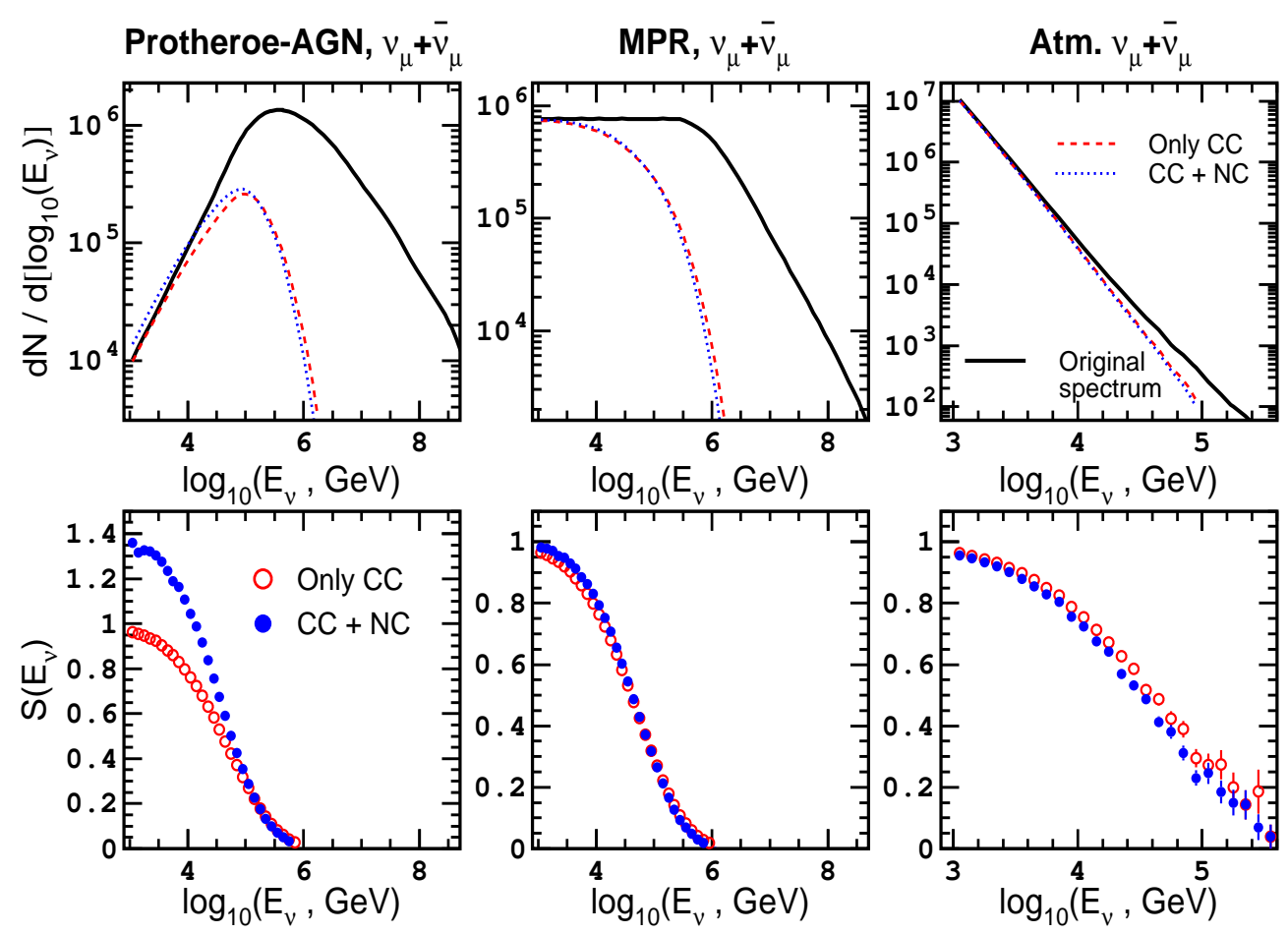

Fig. 4. Upper row: muon neutrino spectra before (solid line) and after propagation through the Earth in the vertical direction with- (dotted lines) and without (dashed lines) accounting for NC interactions. Left: AGN Protheroe spectrum [22]; middle: MPR upper bound on diffuse neutrino flux [23]; right: atmospheric muon neutrino spectrum in [24] including both neutrinos from pion and kaon decay and prompt neutrinos from charmed meson decay (calculated in the Recombination Quark-Parton Model frame). We considered the same amount of neutrinos and anti-neutrinos $\left(\phi_{\nu_{\mu}}: \phi_{\bar{\nu}_{\mu}}=1: 1\right)$. Lower row: the screening factor $S\left(E_{\nu_{\mu}}\right)=\Phi_{1}\left(E_{\nu_{\mu}}\right) / \Phi_{0}\left(E_{\nu_{\mu}}\right)$ computed with fluxes presented in upper plots. Open circles: accounting for CC interactions only; full circles: $\mathrm{MC}$ including both $\mathrm{NC}$ and $\mathrm{CC}$ interactions.

CCs are simulated and when also NCs interactions are accounted for.

We considered the spectrum predicted by Protheroe [22] for diffuse fluxes of neutrinos produced by active galactic nuclei (AGNs), a flux equal to the Mannheim, Protheroe and Rachen (MPR) upper bound [23] and the atmospheric muon neutrino spectrum from [24]. Results are shown in Fig. 4 only for the vertical direction, that is the direction where interactions are most probable given the largest column depth. Results on screening factors (shown in the three lower panels) are in agreement with the results reported in [3], but, as we will see below, large values of screening factors $(S>1)$ cannot be interpreted as a significant enhancement of the emerging neutrino spectrum due to $\mathrm{NC}$ interactions (as it is concluded in [3]). As a matter of fact, this effect is due to the spectral shape. Considering upper panels in Fig. 4 where emerging neutrino spectra are given one can conclude that effects of NC interactions can be summarized in two points. Firstly, NCs result in a slight shift of the mean energy of the spectra emerging after propagation to lower 
energies compared to the spectra obtained simulating only CCs (the spectral shapes are almost the same). This energy shift decreases with increasing nadir angles $\theta$. For the AGN spectrum [22] is equal to $22 \%, 9 \%$ and $3 \%$ for $\theta=0^{\circ}$, $\theta=60^{\circ}$ and $\theta=80^{\circ}$, respectively. The shift is due to the decrease of neutrino energy in NCs since a fraction of the parent neutrino energy is taken away by the hadronic shower, hence the resulting neutrino has lower energy than its parent. Secondly, some enhancement of emerging spectra is observed when $\mathrm{NC}$ interactions are accounted for with respect to the case in which the simulation is performed without NCs. The most remarkable enhancement is found for the AGN spectrum in Ref. [22] for which the ratios of the integral of the emerging spectra with and without including $\mathrm{NC}$ interactions are equal to $1.13,1.02$ and 1.003 for $\theta=0^{\circ}, \theta=60^{\circ}$ and $\theta=80^{\circ}$, respectively. Using the values for $\theta=60^{\circ}(\cos \theta=0.5)$ and this AGN flux to estimate the corresponding effect for the whole lower hemisphere (given that dependencies are more or less linear ones) we obtain a $9 \%$ energy shift in the spectral maximum and a $2 \%$ flux enhancement due to NC interactions. Thus, when NCs are taken into account, neutrino spectra are shifted to lower energies by an amount that hardly will be appreciated by UNTs for which the typical error on neutrino energy measurement is never less than a factor of 2 (see, e.g., [25]). Also the enhancement of neutrino fluxes (and consequently of counting rates) due to NCs is small compared to experimental and simulation uncertainties in UNTs [12-17].

The noticeable increase (up to $S\left(E_{\nu_{\mu}}\right) \approx 1.35$ ) of the screening factor in the left bottom panel of Fig. 4 for the considered AGN spectrum [22] in the TeV range due to $\mathrm{NC}$ interactions is in a good agreement with [3]. But we believe that $S\left(E_{\nu_{\mu}}\right)$ is not the proper variable to estimate the effective enhancement of the emerging spectrum after propagation, and hence of event rates. The effect of NCs can be understood better if we compare the neutrino emerging spectra obtained with and without accounting for NCs. As shown in Fig. 4 (upper panels) these spectra are very similar. Hence, the enhancement in terms of $S\left(E_{\nu_{\mu}}\right)$ is the result only of the slight energy shift of the emerging spectrum after propagation in the Earth relative to the initial spectrum at the Earth surface. The increase on the screening factor is larger for spectra with a maximum and steeply falling down for decreasing energies below the energy corresponding to the maximum (see Protheroe AGN spectrum in Fig. 4). The effect is much smaller for flat spectra or power law spectra with negative spectral index, such is the case for MPR and for atmospheric neutrino spectra.

\section{Conclusions}

We have shown that the effect of NC interactions of ultra and extremely high energy muon and electron neutrinos propagating through the Earth on the 
neutrino flux emerging from the Earth does not play a significant role. Consequently event rates in UNTs, such as AMANDA [12], ANTARES [13], Baikal [14], IceCube [15], NEMO [16], NESTOR [17], are not much affected by NC effects during propagation in the Earth. Their effect can be neglected if the required accuracy of calculations is lower than $\approx 10 \%$ in terms of energy measurement and few percents in terms of absolute event rates. Calculations of counting rates can be much faster if the simple formula in Eq. (1) is used compared to a full propagation of neutrinos through the Earth with MC simulations or semi-analytical methods.

\section{References}

[1] R. Gandhi, Ch. Quigg, M. Reno and I. Sarcevic, Astropart. Phys. 5 (1996) 81. Available from $<$ arXiv:hep-ph/9512364 $>$.

[2] V. A. Naumov and L. Perrone, Astropart. Phys. 10 (1999) 239. Available from $<$ arXiv:hep-ph/9804301 $>$.

[3] J. Kwiecinski, A. D. Martin and A. M. Stasto, Acta Phys. Polon. B31 (2000) 1273. Available from <arXiv:hep-ph/0004109>; A. M. Stasto, Int. J. Mod. Phys. A19 (2004) 317. Available from < arXiv:astro-ph/0310636>.

[4] S. Dutta, M. Reno and I. Sarcevic, Phys. Rev. D62 (2000) 123001. Available from $<$ arXiv:hep-ph/0005310 $>$.

[5] J. F. Beacom, P. Crotty and E. W. Kolb, Phys. Rev. D66 (2002) 021302. Available from $<$ arXiv:astro-ph/0111482 $>$.

[6] S. Bottai and S. Giurgola, Astropart. Phys. 18 (2003) 539. Available from $<$ arXiv:astro-ph/0205325 $>$.

[7] M. Kowalski and A. Gazizov, in: Proceedings of 28th International Cosmic Ray Conference, Tsukuba, 2003, p. 1459. Available from $<$ arXiv:astro-ph/0312202 >; M. Kowalski and A. Gazizov, astro-ph/0406439.

[8] E. Bugaev, T. Montaruli, Yu. Shlepin and I. Sokalski, Astropart. Phys. 21 (2004) 491. Available from < arXiv:hep-ph/0312295>.

[9] Sh. Yoshida, R. Ishibashi and H. Miyamoto, Phys. Rev. D69 (2004) 103004. Available from $<$ arXiv:astro-ph/0312078 $>$.

[10] J. Jones, I. Mocioiu, M. H. Reno and I. Sarcevic, Phys. Rev. D69 (2004) 033004. Available from $<$ arXiv:hep-ph/0308042 $>$.

[11] D. Fargion, P. G. De Sanctis Lucentini and M. De Santis, Astrophys. J. 613 (2004) 1285. Available from <arXiv:hep-ph/0305128 $>$.

[12] AMANDA Collaboration: F. Halzen et al., hep-ex/9611014; M. Ackermann et al., hep-ex/0405035 see URL <http://amanda.uci.edu/>. 
[13] ANTARES Collaboration: E. Aslanides et al., astro-ph/9907432 T. Montaruli et al., in: Proceedings of 28th International Cosmic Ray Conference, Tsukuba, 2003, p.1357. Available from <arXiv:physics/0306057>; see URL $<$ http://antares.in2p3.fr/p.

[14] Baikal Collaboration: I. A. Belolaptikov et al., Astropart. Phys. 7 (1997) 263; R. Wischnewski et al., in: Proceedings of 28th International Cosmic Ray Conference, Tsukuba, 2003, p. 1353. Available from $<$ arXiv:astro-ph/0305302 >; see URL $<$ http://baikal1.jinr.ru/ $>$.

[15] IceCube Collaboration: J. Ahrens et al., astro-ph/0305196 see URL $<$ http://icecube.wisc.edu/ $>$.

[16] NEMO Collaboration: G. Riccobene et al., in: Proceedings of second Workshop on Methodical Aspects of Underwater/Underice Neutrino Telescopes, Hamburg, 2001, p. 61; see URL <http://nemoweb.lns.infn.it/>.

[17] NESTOR Collaboration: S. E. Tzamarias et al., NIM A502 (2003) 150; see URL $<$ http://www.nestor.org.gr/ $>$.

[18] S. L. Glashow, Phys. Rev. 118 (1960) 316.

[19] H. L. Lai et al., Phys. Rev. D51 (1995) 4763. Available from $<$ arXiv:hep-ph/9410404>

[20] H. Plothow-Besch, Comput. Phys. Comm. 75 (1993) 396.

[21] A. Dziewonski, Encyclopedia of Solid Earth Geophysics, ed. by D. E. James (Van Nostrand Reinhold, New York, 1989), p.331, we used the formula from this work cited in [1].

[22] R. J. Protheroe, in Accretion Phenomena and Related Outflows, ed. Wickramasinghe et al. (Astronomical Society of the Pacific, San Francisco, 1997).

[23] K. Mannheim, R. J. Protheroe and J. P. Rachen, Phys. Rev. D63 (2001) 023003. Available from $<$ arXiv:astro-ph/9812398 $>$.

[24] V. A. Naumov, in: Proceedings of second Workshop on Methodical Aspects of Underwater/Underground Telescopes, Hamburg, 2001, p. 31. Available from $<$ arXiv:hep-ph/0201310 $>$.

[25] I. Sokalski et al., Phys. Atom. Nucl. 67 (2004) 1172. Available from $<$ arXiv:astro-ph/0310130>. 